\title{
A Case of Candida albicans Endophthalmitis with No Predisposing Risk Factors and a Distant Source of Infection
}

\author{
Randhir Chavan Mohammad Z. Mustafa \\ Nirodhini Narendran Shoaib Tarin Yit Yang \\ Wolverhampton Eye Infirmary, New Cross Hospital, Wolverhampton, UK
}

\section{Key Words}

Candida albicans · Endophthalmitis · Amphotericin B · Voriconazole

\begin{abstract}
Purpose: To report a case of Candida albicans endophthalmitis with no identifiable predisposing risk factors.

Case Report: A 57-year-old male presented with a 3-day history of worsening floaters and reduced visual acuity. Fundoscopy and optical coherence tomography showed presence of fluffy white preretinal and intraretinal infiltrates. With no past medical history or evidence of immunosuppression but having travelled abroad and suffered from diarrhoea, fungal aetiology was thought to be unlikely and as a result, treatment was commenced for toxoplasma. Despite treatment, his vision did not improve. Initial investigations including inflammatory markers, serology for toxoplasmosis, blood culture, chest radiograph and aqueous sampling could not identify a source of infection. However, polymerase chain reaction results from vitreous sampling revealed $C$. albicans. As a result, the patient was treated with intravenous voriconazole and intravitreal amphotericin B. As initial clinical improvement was limited, a vitrectomy was performed with further intravitreal amphotericin B. Clinical improvement was rapid following vitrectomy. After repeated Gram staining and culture of infected toenails, Gram-positive yeast cells were isolated.

Conclusion: Although C. albicans is a frequent cause of endogenous endophthalmitis, patients often have one or more predisposing systemic condition assisting the diagnosis. The present case illustrates that (1) even in the absence of any predisposing risk factors, $C$. albicans should be considered as a possible differential diagnosis in recalcitrant uveitis, and (2) endogenous candida endophthalmitis can be a result of fungal infections from distant sites such as the toenails.
\end{abstract}




\section{Introduction}

Candida endophthalmitis is a potentially devastating ocular condition. It presents as a complication in $9-45 \%$ of all cases of candidaemia (commonly with Candida albicans), associated with well-known predisposing risk factors such as intravenous drug abuse, immunodeficiency, prolonged systemic corticosteroid or antibiotic therapy, cytotoxic chemotherapy and long-term parenteral feeds $[1,2]$. In rare cases of endogenous infection with no presdisposing risk factors, diagnosis and justification for use of intravitreal antifungal agents can be difficult [3].

This is a case report describing the management of a patient with endogenous endophalmitis due to $C$. albicans which was not associated with any of the known predisposing factors of candidaemia. The report demonstrates the development of a differential diagnosis in an atypical scenario, the eventual confirmation of the infective agent from vitreous sampling and also the identification of the possible source of the Candida organism leading to the justification for an aggressive antifungal regimen which resulted in a good outcome.

\section{Case Report}

A 57-year-old male presented with a 3-day history of blurred vision, floaters and reduced visual acuity to $6 / 9$ in his left eye. His right eye was asymptomatic with a visual acuity of 6/5. Ocular examination of the left eye showed inflammatory activity in the anterior and posterior segments with mild anterior uveitis and vitritis along with several fluffy white retinal infiltrates near the foveal centre (fig. 1). The right eye was completely normal. An optical coherence tomography (OCT) scan confirmed the presence of vitreous and intraretinal infiltrates (ig. 2).

Further clinical evaluation revealed that the patient had been fit and well but had a brief episode of diarrhoea several weeks earlier whilst visiting the Canary Islands, Spain, and he also had developed signs of toenail infection affecting several toes over the previous 6 months. He was not on any medication and there was no history of other illness, intravenous drug abuse or any opportunistic infections to suggest immunosuppression. Clinical suspicion at this stage was of infective retinitis due to fungal infection or toxoplasmosis. Because the patient was not immunocompromised, a fungal aetiology was felt to be less likely and he was commenced on oral azithromicin $500 \mathrm{mg}$ once a day and hourly topical dexamethasone $0.1 \%$.

As this was an empirical treatment, an extensive uveitis screen was performed, but all of these initial tests were normal or negative, including chest X-ray, haematological profile, ESR, CRP, serum ACE level, serology for toxoplasma and VDRL as well as HIV. Polymerase chain reaction (PCR) analysis of aqueous was also negative for toxoplasma, CMV, VZV, HSV, Mycobacterium, Candida and fungal RNA. Blood cultures and cultures of nail clippings from his infected toenails were negative.

After 1 week of follow-up, the patient had worsened considerably with increasing vitritis and vitreous infiltrates at the vitreoretinal interface causing a reduction in vision to 6/60 (fig. $1 \mathrm{~b}$ ). At this point, the decision was made to carry out diagnostic vitreous biopsy. An automated vitrector was used to obtain a vitreous biopsy and samples were sent for PCR analysis and microbiological testing. From this sample, PCR analysis was positive for C. albicans DNA, which was confirmed several weeks later by a positive culture.

Following confirmation of Candida DNA on PCR analysis of the vitreous sample, intravenous voriconazole $400 \mathrm{mg}$ b.d. and intravitreal amphotericin B $(5 \mu \mathrm{g} / 0.1 \mathrm{ml})$ was immediately commenced. After 7 days of systemic antifungal therapy with the above regimen, there was very little treatment response and hence a 3-port pars plana vitrectomy was performed and intravitreal administration of amphotericin B $(5 \mu \mathrm{g} / 0.1 \mathrm{ml})$ was repeated. Diagnostic sampling was repeated on this occasion and although PCR analysis was negative for Candida DNA from the second vitrectomy sample, culture yielded positive growth and histopathological analysis showed typical yeast-like organisms with the 
classical budding pattern appearance (fig. 3 ). The further confirmation of Candida on a second vitreous biopsy justified continuing antifungal therapy with oral voriconazole (200 mg b.d.).

The confirmation of the source of the endogenous candida endophthalmitis came much later when repeated samples of nail clippings eventually detected Gram-positive yeast cells 30 days after the initial presentation.

The patient improved following the vitrectomy and there were no signs of vitreous infiltrates (fig. 1c) after 1 week and OCT showed minimal residual intraretinal oedema (fig. 2b). After further 2 months of follow-up, his vision had improved to $6 / 9$ with complete resolution of retinal oedema.

\section{Discussion}

The diagnostic approach in this case was influenced by a strong, pretest clinical suspicion of an underlying infective aetiology due to the absence of systemic inflammatory signs, cellular infiltration in both anterior and posterior segments, unilateral involvement and the presence of focal infiltration in the posterior vitreous and anterior retinal layers [4].

The organisms involved in cases of infective uveitis depend largely on whether the patient is immunocompromised or immunocompetent. Fungal and infections by other opportunistic pathogens are much more likely to occur in immunocompromised individuals [3].

C. albicans is the most common cause of endogenous endophthalmitis and frequently presents as a unilateral posterior uveitis associated with fluffy white vitreous and retinal infiltrates which have often been described as a 'string of pearls' appearance. It almost always occurs in patients who are immunocompromised or have other predisposing risk factors such as intravenous drug abuse [2,3]. Although our patient had a very typical fundus appearance suggestive of fungal endophthalmitis, it was very unusual in that he was immunocompetent and had no apparent risk factors. In the world literature, there are only two case reports of endogenous candida endophthalmitis with no previous predisposing risk factors $[5,6]$. The first case was a patient with onychomycosis and vaginal candidiasis [5]. The culture for the same strain of Candida was positive for the nail and the eye. The second case had used inhaled steroids for asthma and could have developed asymptomatic oropharyngeal candidiasis [6]. From these reported cases and the experience in our case, it appears that endogenous candida endophthalmitis could occur from mild fungal infections from a distant site such as the toenail.

Another interesting and unexpected finding in this case was the negative PCR result from the vitreous sample taken at the second vitrectomy when this specimen eventually grew Candida on culture. This paradoxical disconnect between PCR and culture results has been reported before, especially in association with repeated sampling. Harper et al. [7] analysed all PCR results of aqueous and vitreous samples in diagnosis of infectious uveitis and concluded that specimens from the first week of presentation (acute stage) were more likely to have positive PCR results than those sampled later. Harper et al. [7] postulate polymorphism and specimen degradation as possible causes of false-negative results. In our case, two vitreous samples were performed. The first yielded positive PCR, but a positive growth on culture took over 3 weeks by which time the second vitrectomy had been performed. The second vitreous 
sample yielded a negative PCR, but yeast-like organisms were identifiable on histopathology and growth was also positive on culture. This illustrates that confirmation of Candida as the causative agent can be difficult as PCR and Gram stain can be falsely negative and culture can take several weeks to yield any diagnostic growth.

Reported cases of endogenous endophthalmitis due to C. albicans tend to have a poor visual outcome [8]. Our case had a good visual outcome despite the infiltrate being located very close to the fovea. The factors contributing to the good outcome in this case were probably the early intervention with a second vitrectomy and intravitreal amphotercin $B$, the continuation of prolonged course of oral voriconazole and also the immunocompetent status of our patient. Systemic antifungal drugs such as fluconazole, voriconazole and amphotericin B given intravenously or orally are indicated in disseminated candidaemia. We chose to use voriconazole as a first-line antifungal for its broad spectrum activity as we did not have confirmation of Candida on the vitreous culture initially and blood cultures were negative. Several reports have suggested early vitrectomy, especially in lesions affecting the macula or significant vitritis. Advantages are thought to be reduction of infectious load, increase in intraocular diffusion of systemically administered medication and reduction of the risk of retinal detachment by limiting vitreoretinal traction $[9,10]$. For these multiple potential benefits, we felt it was justified to perform a vitrectomy and allowed the intravitreal administration of amphotericin $B(5 \mu \mathrm{g} / 0.1 \mathrm{ml})$ which tends to have poor ocular penetration when given systemically $[11,12]$.

In summary, this interesting case illustrates that endogenous endophthalmitis due to C. albicans is rare but can occur in patients with no predisposing risk factors or evidence of candidaemia. In these situations, when the clinical findings suggest a fungal aetiology, repeated diagnostic testing with a combination of PCR, Gram staining and culture of vitreous and suspected primary sites of infection can be helpful in confirming the definitive diagnosis and provide confidence for adherence to a prolonged course of systemic antifungal therapy to achieve eradication of the organism and a good visual outcome.

\section{Acknowledgement}

The authors are grateful for a medical educational grant from Novartis Pharmaceuticals to cover publication costs in full. 

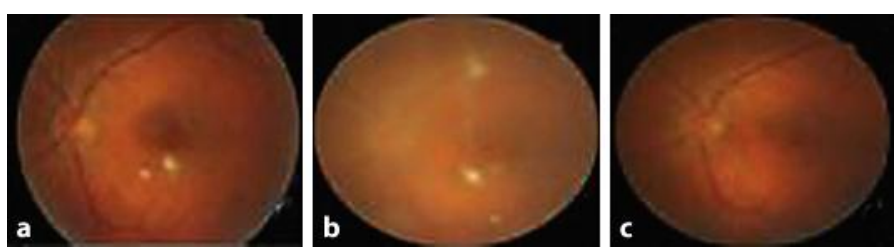

Fig. 1. Fundus photographs of the patients' left eye showing fluffy white retinal and vitreous infiltrates at presentation (a), 1 week after presentation (b) and after surgery (c).

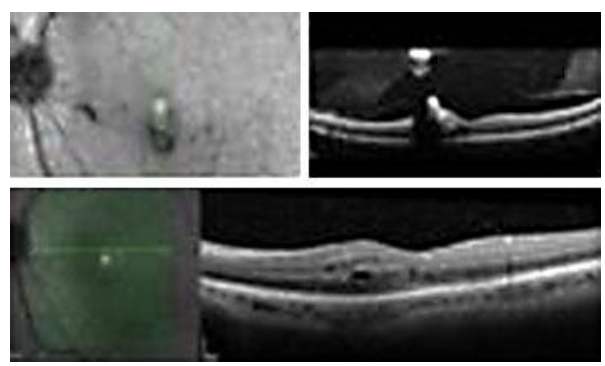

Fig. 2. OCT of the patients' left eye showing intraretinal, preretinal and vitreous infiltrates with vitritis (top). One week following vitrectomy, no retinal or vitreous infiltrates and presence of residual intraretinal oedema were observed (bottom).

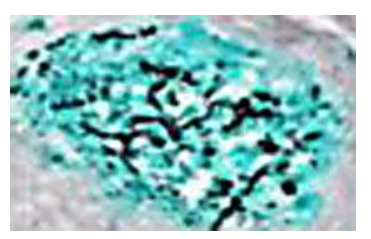

Fig. 3. Histopathology of the vitreous specimen stained with methenamine silver showing yeast with budding pattern. 


\section{References}

1 Riddell J 4th, Comer GM, Kauffman CA: Treatment of endogenous fungal endophthalmitis: focus on new antifungal agents. Clin Infect Dis 2011;52:648-653.

-2 Sallam A, Taylor SR, Khan A, McCluskey P, Lynn WA, Manku K, Pacheco PA, Lightman S: Factors determining visual outcome in endogenous candida endophthalmitis. Retina 2012;35:1129-1134.

-3 Hamza HS, Lowenstein A, Haller JA: Fungal retinitis and endophthalmitis. Ophthalmology Clinics of North America 1999;12:89-108.

4 Nussenblatt RB, Whitcup SM: Development of a differential diagnosis; in Nussenblatt RB, Whitcup SM (eds): Uveitis - Fundamentals and Clinical Practice, ed 3. Philadelphia, Mosby (Elsevier), 2004, pp 6675.

5 Kostick DA, Foster RE, Lowder CY, Meyers SM, McHenry ME: Endogenous endophthalmitis caused by Candida albicans in a healthy woman. Am J OphthalmoI 1992;113:593-594.

-6 Stanbury RM, Chignell AH, Graham EM: Endogenous Candida endophthalmitis with no apparent predisposing factors. Eye 1998;12:321-323.

7 Harper TW, Miller D, Schiffman JC, Davis JL: Polymerase chain reaction analysis of aqueous and vitreous specimens in the diagnosis of posterior segment infectious uveitis. Am J Ophthalmol 2009;147:140-147.

$>8$ Schiedler V, Scott IU, Flynn HW Jr, Davis JL, Benz MS, Miller D: Culture-proven endogenous endophthalmitis: clinical features and visual acuity outcomes. Am J Ophthalmol 2004;137:725-731.

-9 O'Day DM, Head WS, Robinson RD, Stern WH, Freeman JM: Intraocular penetration of systemically administered antifungal agents. Curr Eye Res 1985;4:131-134.

-10 Louie A, Liu W, Miller DA, Sucke AC, Liu Q-F, Drusano GL, Mayers M, Miller MH: Efficacies of high-dose fluconazole plus amphotericin B and high-dose fluconazole plus 5 -fluorocytosine versus amphotericin $B$ fluconazole, and 5-fluorocytosine monotherapies in treatment of experimental endocarditis, endophthalmitis, and pyelonephritis due to Candida albicans. Antimicrob Agents Chemother 1999;43:2831-2840.

11 Takebayashi H, Mizota A, Tanaka M: Relation between stage of endogenous fungal endophthalmitis and prognosis. Graefes Arch Clin Exp Ophthalmol 2006;244:816-820.

-12 Sarria JC, Bradley JC, Habash R, Mitchell KT, Kimbrough RC, Vidal AM: Candida glabrata endophthalmitis treated successfully with caspofungin. Clin Infect Dis 2005;40:e46-e48.

This case report was presented at the UK National Retinal Case Awards Event, City Hospital, Birmingham, UK, on February 22, 2012, sponsored by Novartis Pharmaceuticals Ltd., UK. 